\title{
Translation Group and Modular Automorphisms for Local Regions
}

\author{
H.-J. Borchers \\ Institut für Theoretische Physik, Universität Göttingen, Bunsenstrasse 9, d-3400 Göttingen, \\ Federal Republic of Germany
}

Dedicated to Res Jost and Arthur Wightman

\section{Introduction}

In this paper we will look at quantum field theory in the setting of Araki, Haag, and Kastler. The system of local observables will be denoted by $\left\{\mathscr{A}(O), \mathscr{A}, \alpha, \mathbf{R}^{d}\right\}$. For details see Sect. II. We are interested in positive energy representations, denoted by $\left\{\pi, U(a), V^{+}, \mathscr{H}\right\}$. In this situation every vector analytic for the energy has the Reeh Schlieder [9] property, which means that this vector is cyclic and separating for the algebras $\pi(\mathscr{A}(O))$ whenever $O$ is a bounded region. As a consequence we find that there exists a modular automorphism $\sigma_{t}$ associated to every pair $\left\{\pi(\mathscr{A}(O))^{\prime \prime}, \Omega\right\}$.

Inspired by statistical mechanical examples one might think that the generators of the modular automorphism group are connected with the energy belonging to the region we consider. On first sight, however, there seems to be no connection between the space-time-translations and the modular automorphisms. Therefore, Buchholz and Junglas [5] have used the Hamiltonian of the vacuum sector in order to construct K.M.S. states for the theory of local observables. In order to obtain a kind of local energy $H^{o}$ such that $\exp \left\{-\beta H^{o}\right\}$ is trace class they had to work with the nuclearity condition of Buchholz and Wichmann [6]. Although this local Hamilton operator had some properties similar to the modular operator, they failed to establish a connection between these objects. This is because their "local" Hamiltonian does not generate a group of automorphisms for the local region.

Recently Buchholz, D'Antoni, and Longo [4] found estimates suggesting that, after rescaling, the modular operator of the double cone tends to the Hamilton operator if the double cone tends to the whole space. In the last section we will discuss a possible procedure proving this result.

We want to show that the space-time translations and the modular automorphisms, indeed, are closely related. Using the Malgrange Zerner theorem it easily shows that expressions of the form

$$
\left(\Omega, \alpha_{a}(A) \sigma_{t}(B)\right)=: E(a, t)
$$

are the boundary value of an analytic function holomorphic in the variables $(a, t)$ provided $\alpha_{a}$ represents the space-time translations, $\sigma_{t}$ the modular automorphisms, 
and $\Omega$ is a vector with compact energy support. Using locality we will show that the function $E(a, t)$ can be analytically extended into a larger domain and that this extension is periodic in $t$ along the imaginary axis.

This paper is organized as follows: In Sect. II we describe the assumptions and notations. In the third section we connect the space-time translations and the modular automorphisms. Since the reason for this close connection is the fact that the translations act locally one can generalize these ideas to other groups which act locally. One example is the Bisognano Wichmann case, where the modular group of the wedge domain acts locally, provided the cyclic and separating vector is the vacuum. This situation will be investigated in Sect. IV. In the last section we discuss in detail the situation described in the beginning taking account the results obtained in Sect. III and IV.

\section{Assumptions and Notations}

In this paper we are dealing with a theory of local observables, a so-called Araki, Haag, Kastler theory. This is a quantum field theory described in terms of bounded operators. To every bounded open region $O$ in $\mathbf{R}^{d}$ is associated a $C^{*}$-algebra $\mathscr{A}(O)$. The $C^{*}$ inductive limit of the increasing family $\{\mathscr{A}(O)\}$ is denoted by $\mathscr{A}$. Furthermore we have the translation group $\mathbf{R}^{d}$ acting on $\mathscr{A}$ as automorphism such that $\alpha_{a} \mathscr{A}(O)=\mathscr{A}(O+a)$ holds for $a \in \mathbf{R}^{d}$. If two domains $O_{1}$ and $O_{2}$ are spacelike separated then we assume, as usual, that $\mathscr{A}\left(\mathrm{O}_{1}\right)$ and $\mathscr{A}\left(\mathrm{O}_{2}\right)$ commute elementwise with each other. Such a system will be denoted by

$$
\left\{\mathscr{A}(O), \mathscr{A}, \alpha, \mathbf{R}^{d}\right\} \text {. }
$$

By $\mathbf{R}^{d}$ we always mean the Minkowski space with points $\left\{x_{0}, \vec{x}\right\}$ and scalar product $(x, y)=x_{0} y_{0}-(\vec{x}, \vec{y})$, where $(\vec{x}, \vec{y})$ is the usual Euclidean scalar product. Therefore $d$ fulfills the restriction $d \geqq 2$.

If $V \subset \mathbf{R}^{d}$ is an open but unbounded domain then $\mathscr{A}(V)$ will be the smallest sub $C^{*}$-algebra of $\mathscr{A}$ containing all $\mathscr{A}(O)$ provided $O \subset V$.

In the triple $\{\pi, U(a), \mathscr{H}\}$ denotes $\mathscr{H}$ a Hilbert space, $\pi$ a non-degenerated representation of the algebra $\left\{\mathscr{A}(O), \mathscr{A}, \alpha, \mathbf{R}^{d}\right\}$ on $\mathscr{H}$ and $U(a)$ continuous unitary representation of the translation group $\mathbf{R}^{d}$ implementing the automorphisms $\alpha_{a}$, i.e.

$$
\pi\left(\alpha_{a} A\right)=U(a) \pi(A) U^{*}(a), \quad a \in \mathbf{R}^{d}, \quad A \in \mathscr{A} .
$$

The open forward light-cone will be denoted by $V^{+}$. A representation $\{\pi, U(a), \mathscr{H}\}$ will be called a representation with spectrum condition if the spectrum of $U(a)$ is contained in the forward light-cone, i.e.

$$
\operatorname{spec} U(a) \subset \overline{V^{+}}
$$

where the bar over a set denotes the closure of this set. Representations with spectrum condition will be denoted by

$$
\left\{\pi, U(a), V^{+}, \mathscr{H}\right\} .
$$


If $U(a)$ is a continuous unitary representation of the translation group on the Hilbert space $\mathscr{H}$, then a vector $\psi \in \mathscr{H}$ is called entire analytic for $U(a)$ if the vector valued function $U(a) \psi$ defined on $\mathbf{R}^{d}$ has an extension as entire analytic vector valued function defined on $\mathbf{C}^{d}$. If $\left\{\pi, U(a), V^{+}, \mathscr{H}\right\}$ is a representation fulfilling spectrum condition, and if $\psi$ is an entire analytic vector for $U(a)$ then the vector valued function

$$
U(a) \pi(A) U^{-1}(a) \psi=\pi\left(\alpha_{a} A\right) \psi
$$

is the boundary value of a vector valued analytic function $F(z)$ which is holomorphic in the forward tube $T^{+}$:

$$
T^{+}=\left\{z \in \mathbf{C}^{d} ; \mathfrak{I} m z \in V^{+}\right\} .
$$

Let $\left\{\pi, U(a), V^{+}, \mathscr{H}\right\}$ be a representation with spectrum condition of the theory of local observables, and let $\psi$ be an entire analytic vector for $U(a)$, then under mild condition on $\pi(\mathscr{A}(O))$ [2] one has the validity of the Reeh Schlieder Theorem [9] for the vector $\psi$ which means that $\psi$ is a cyclic vector for the algebra $\pi(\mathscr{A}(O))$. Since $O$ is bounded, the locality condition implies that this vector is also separating. From this we conclude that we can use Tomita's theory of modular Hilbert algebras $[10,11]$ for the von Neumann algebra generated by $\pi(\mathscr{A}(O))$. Before discussing this we need some more notations and concepts used in the theory of local observables.

If $V$ is an open set in $\mathbf{R}^{d}$ then we denote by $V^{\prime}$ the following set:

$$
V^{\prime}=\text { int }\{x ; x-y \text { is spacelike for every } y \in V\} \text {. }
$$

Here int $\{$.$\} denotes the interior of the set in the bracket. A set W$ is called a wedge domain, if in a suitable Lorentz frame it is of the form:

$$
W=\left\{x ;\left|x_{0}\right|< \pm x_{1}\right\} \text {. }
$$

A set $D$ is called a double cone if it is of the form $\left\{a+V^{+}\right\} \cap\left\{b-V^{-}\right\}$. In order that this set is not empty we must have $b \in a+V^{+}$. If we have to distinguish between different double cones we write $D(a, b)$.

Sometimes one wants the commutant of a localized algebra to be also localized. If this is the case for a suitable family of sets then we are calling such a representation a representation with duality property.

II.1. Definition. Let $\left\{\mathscr{A}(O), \mathscr{A}, \alpha, \mathbf{R}^{d}\right\}$ be a theory of local observables. Let $\{\pi, U(a), \mathscr{H}\}$ be a representation of this theory. We are saying $\{\pi, U(a), \mathscr{H}\}$ has the duality property. If one has the relation

$$
\pi(V)^{\prime}=\pi\left(V^{\prime}\right)^{\prime \prime}
$$

when $V$ is taken from the following family $\mathscr{F}$ of sets,

$\mathscr{F}$ contains: wedges, double cones, and spacelike complements of double cones.

It should be remarked that we are not using the most general form of the duality property, because one could enlarge the above family of sets. Furthermore one should be aware of the fact that the duality property is not a property of the algebra, but a property of representations. 
If $\left\{\pi, U(a), V^{+}, \mathscr{H}\right\}$ is a representation of the theory of local observables then for any region $V$ the von Neumann-algebra $\mathscr{M}(V)$ is the von Neumann-algebra generated by $\pi(\mathscr{A}(V))$,

$$
\mathscr{M}(V)=\pi(\mathscr{A}(V))^{\prime \prime} .
$$

If $\Omega$ is a vector entire analytic for $U(a)$ and if $V^{\prime} \neq \emptyset$ then $\Omega$ is a cyclic and separating vector for $\mathscr{M}(V)$. Hence by Tomita's theory of modular Hilbert algebras there exists a modular operator $\Delta$ with $\Delta \Omega=\Omega$ defining the modular automorphism group $\sigma_{t}: \mathscr{M}(V) \rightarrow \mathscr{M}(V)$ given by the formula

$$
\sigma_{t}(A)=\Delta^{t t} A \Delta^{-\imath t}, \quad A \in \mathscr{M}(V) .
$$

In addition there exists a modular conjugation $J$ which is an antilinear operator with $J^{2}=1$ such that $j(A):=J A J \in \mathscr{M}(V)^{\prime}$ for every $A \in \mathscr{M}(V)$. The modular conjugation $J$ has $\Omega$ a fixed point, i.e.: $J \Omega=\Omega$. The modular conjugation and the modular operator are connected by the equation $J \Delta^{\frac{1}{2}} A \Omega=A^{*} \Omega$ for every $A \in \mathscr{M}(V)$. Consequently $\sigma_{t}(A) \Omega=\Delta^{t t} A \Omega$ is not only defined for real $t$ but also for $t$ having an imaginary part restricted to $-\frac{1}{2} \leqq \mathfrak{I} m t \leqq 0$. From this we conclude that the vector valued function $\sigma_{t}(A) \Omega$ is analytic in the strip $S\left(-\frac{1}{2}, 0\right)$, where

$$
S(a, b)=\{z \in \mathbf{C} ; a<\mathfrak{I} m z<b\} .
$$

Since ad $\Delta^{i t}$ maps $\mathscr{M}(V)$ onto $\mathscr{M}(V)$ it is also true for $\mathscr{M}(V)^{\prime}$. Therefore one can define the automorphism $\sigma_{t}$ also on the algebra $\mathscr{M}(V)^{\prime}$. Usually however, one identifies $\sigma_{t}$ on $\mathscr{M}(V)^{\prime}$ with ad $\Delta^{-t t}$. The reason for this is the relation $J \Delta J=\Delta^{-1}$. So with the standarad choice one obtains $j\left(\sigma_{t}(A)\right)=\sigma_{t}(j(A))$ for $A \in \mathscr{M}(V)$ or $A \in \mathscr{M}(V)$. Consequently one concludes that for $B \in \mathscr{M}(V)^{\prime}$ the expression $\sigma_{t}(B) \Omega$ is analytic in the strip $S\left(0, \frac{1}{2}\right)$.

\section{Locality and Periodicity}

In this section let $\left\{\mathscr{A}(O), \mathscr{A}, \alpha, \mathbf{R}^{d}\right\}$ be a theory of local observables, $\left\{\pi, U(a), V^{+}, \mathscr{H}\right\}$ a representation fulfilling spectrum condition and $\Omega \in \mathscr{H}$ be a vector entire analytic for $U(a)$. Let $V \subset \mathbf{R}^{d}$ such that $V^{\prime}$ is not empty and let $D$ be a double cone.

III.1. Definition. For $A \in \mathscr{M}(V)$ and $B \in \mathscr{M}(D)$ and $\sigma_{t}$ the modular automorphism of $\{\mathscr{M}(V), \Omega\}$ define

$$
\begin{aligned}
E(a, t) & =\left(\Omega . \alpha_{a}(B) \sigma_{t}(A) \Omega\right), \\
F(a, t) & =\left(\Omega, \sigma_{t}(A) \alpha_{a}(B) \Omega\right), \\
t & \in \mathbf{R} \quad a \in \mathbf{R}^{d} .
\end{aligned}
$$

If needed we write $E_{A, B}(a, t)$ and $F_{A, B}(a, t)$ which implies $E_{A, B}(a, t)$ $=\overline{F_{A^{*}, B^{*}}(a, t)}$. Both these functions are boundary values of analytic functions. 
III.2. Lemma. (a) The function $E(a, t)$ is the boundary value of an analytic function $E(z, \tau)$ holomorphic in

$$
T^{-} \times S\left(-\frac{1}{2}, 0\right) \text {. }
$$

(b) The function $F(a, t)$ is the boundary value of an analytic function $F(z, \tau)$ holomorphic in

$$
T^{+} \times S\left(0, \frac{1}{2}\right) \text {. }
$$

(c) In both cases the boundary value is taken in the sense of continuous functions.

Proof. This is a consequence of the properties of the vector valued functions $\sigma_{t}(A) \Omega$ and $\alpha_{a}(B) \Omega$ explained in Sect. II.

We now introduce the following two sets, which are not empty if $D$ is sufficiently small.

III.3. Definition. Put

$$
\begin{aligned}
& G_{1}=\operatorname{int}\{a ; a+D \subset V\}, \\
& G_{2}=\operatorname{int}\left\{a ; a+D \subset V^{\prime}\right\} .
\end{aligned}
$$

With this notation we obtain:

III.4. Theorem. Under the assumptions made earlier in this section one has: $E(z, \tau)$ and $F(z, \tau)$ are two different branches of one holomorphic function $H(z, \tau)$, which is periodic in $\tau$ in pure imaginary direction with period $i$,

$$
H(z, \tau+\imath)=H(z, \tau)
$$

whenever $(z, \tau)$ is in the domain of holomorphy of $H(z, \tau)$.

In particular one has

$$
\begin{array}{lll}
E(z, \tau)=H(z, \tau), & \text { for } \quad(z, \tau) \in T^{-} \times S\left(-\frac{1}{2}, 0\right), \\
E(z, \tau)=H(z, \tau), & \text { for } \quad(z, \tau) \in T^{+} \times S\left(0, \frac{1}{2}\right) .
\end{array}
$$

The different branches are connected by means of the edge of the wedge theorem [3]. One has

$$
\begin{aligned}
& E(a, t)=F(a, t), \quad \text { for } \quad a \in G_{2}, \quad t \in \mathbf{R}, \\
& E\left(a, t-\frac{l}{2}\right)=F\left(a, t+\frac{l}{2}\right), \quad \text { for } \quad a \in G_{1}, \quad t \in \mathbf{R} .
\end{aligned}
$$

Proof. If $a \in G_{2}$ then $a+D \subset V^{\prime}$, hence $\alpha_{a}(B) \in \mathscr{M}\left(V^{\prime}\right)$. Therefore, it commutes with $\sigma_{t}(A) \in \mathscr{M}(V)$. This gives $E(a, t)=F(a, t)$ for $a \in G_{2}$.

For computing $E\left(a, t-\frac{l}{2}\right)$ we make use of the equation $J \Delta^{\frac{1}{2}} A \Omega=A^{*} \Omega$. Hence we obtain

$$
\begin{aligned}
E\left(a, t-\frac{l}{2}\right) & =\left(\Omega, \alpha_{a}(B) \Delta^{\frac{1}{2}} \sigma_{t}(A) \Omega\right) \\
& =\left(\Omega, \alpha_{a}(B) J \sigma_{t}\left(A^{*}\right) \Omega\right) \\
& =\left(\Omega, \alpha_{a}(B) j\left(\sigma_{t}\left(A^{*}\right)\right) \Omega\right) .
\end{aligned}
$$


On the other hand one has

$$
\begin{aligned}
F\left(a, t+\frac{l}{2}\right) & =\left(\Omega, \sigma_{t}(A) \Delta^{1 / 2} \alpha_{a}(B) \Omega\right) \\
& =\left(\Delta^{1 / 2} \sigma_{t}\left(A^{*}\right) \Omega, \alpha_{a}(B) \Omega\right) \\
& =\left(J \sigma_{t}(A) \Omega, \alpha_{a}(B) \Omega\right) \\
& =\left(j\left(\sigma_{t}(A)\right) \Omega, \alpha_{a}(B) \Omega\right) \\
& =\left(\Omega, j\left(\sigma_{t}\left(A^{*}\right)\right) \alpha_{a}(B) \Omega\right)
\end{aligned}
$$

Remark that $j\left(\sigma_{t}\left(A^{*}\right)\right)$ belongs to $\mathscr{M}(V)^{\prime}$ and $\alpha_{a}(B)$ belongs to $\mathscr{M}(V)$ for $a \in G_{1}$. This gives the stated two connections between the functions $E$ and $F$. This in turn implies the other statements of the theorem.

We end this section with a remark on the K.M.S. conditions, which is one characteristic in the theory of modular Hilbert algebras. If $a \in G_{1}$ then $\alpha_{a}(B)$ belongs to $\mathscr{M}(V)$. In this case the expression $\left(\Omega, \alpha_{a}(B) \Delta^{\lambda} \sigma_{t}(A) \Omega\right)$ is defined and continuous for $0 \leqq \lambda \leqq 1$ and analytic in $\lambda+t$ for $0<\lambda<1$.

If on the other hand we fix $\lambda$ to be $1 / 2$ then $\Delta^{\frac{1}{2}} \sigma_{t}(A) \Omega$ is defined and consequently $\left(\Omega, \alpha_{a}(B) \Delta^{\frac{1}{2}} \sigma_{t}(A) \Omega\right)$ possesses in $a$ an analytic continuation into the tube $T^{-}$. This means the following properties for the function $E\left(a, t-\frac{l}{2}\right)$ :

(a) For $a \in G_{1}$ it has an analytic continuation in $t$ into the strip $-1 / 2<\mathfrak{I} m t<0$.

( $\beta$ ) For $t \in \mathbf{R}$ the function $E\left(a, t-\frac{l}{2}\right)$ has in $a$ an analytic continuation into the tube $T^{-}$.

From this we conclude by the Malgrange-Zerner theorem that $E\left(a, t-\frac{l}{2}\right)$ has a continuation in both variables in some suitable subset of $\left\{a \in T^{-}\right\} \times\left\{t \in S\left(-\frac{1}{2}, 0\right)\right\}$, which contains $\left\{a \in G_{1}\right\} \times\left\{t \in S\left(-\frac{1}{2}, 0\right)\right\}$ as a limiting subset. Therefore, we can conclude that $E(a, t)$ has an analytic continuation into some subset of $T^{-} \times\{t \in S(-1,0)\}$. On the other hand we know that for $a \in G_{1}$ the relation $E\left(a, t-\frac{l}{2}\right)=F\left(a, t+\frac{l}{2}\right)$ holds. Consequently, the continuation of $E\left(a, t-\frac{l}{2}\right)$ coincides with the continuation of $F\left(a, t+\frac{l}{2}\right)$. Hence for $a \in G_{1}$ we have $E(a, t-\imath)=F(a, t)$. Expressing this in terms of expectation values, we obtain exactly the K.M.S. boundary condition.

\section{The Case of Two Modular Automorphisms}

Looking at the derivations and the results of the last section, we see that one property was essential, namely that translations act locally, which means that it sends the algebra associated with a bounded region onto the algebra of another bounded region. Therefore we would expect similar behaviour of expectation values if we replace the translations by another local transformation. This idea shall 
be exploited for the case where the translation is replaced by the modular automorphism group of some bigger region, which will be the wedge domain.

If one starts with a Wightman field theory of a finite number of fields and assumes that the whole Lorentz group acts on this theory, one can determine the modular group and the modular involution for the wedge region, provided we can pass from the Wightman fields to the algebra of local observables. These results are due to Bisognano and Wichmann [1]. They showed that the modular transformations are Lorentz boosts having the wedge invariant and that modular involution is composed by the time reflection and a reflection of the space at the hypersurface (defining the wedge) such that the wedge is mapped to spacelike complement. Here the vacuum vector is used as cyclic and separating vector. The physically very interesting outcome of these investigations is that the modular transformations and the modular involution are local transformations based on point-transformations of the basespace $\mathbf{R}^{d}$.

There is only one other situation known where one has the same strong information for the modular automorphisms. This is the case of a massless free field where the domain in question is the double cone. This result is due to Hislop and Longo [8].

Leaving the last case aside we will look at the situation where the large region is the wedge region. We furthermore assume that we have a vector $\Omega$ which is cyclic and separating not only for the wedge region $W$ but also for a subregion $O \subset W$. In addition we assume that the modular automorphism $\sigma_{t}^{W}$ of the pair $\{\mathscr{M}(W), \Omega\}$ is a continuous point-transformation, i.e.

$$
\sigma_{t} \mathscr{M}(O)=\mathscr{M}\left(O_{\sigma_{t}}\right)
$$

This means we have a continuous group of homeomorphisms of the wedge region $y \rightarrow y(t), y, y(t) \in W$ such that $O_{\sigma_{t}}=\{y(t) ; y \in O\}$. $I \subset \mathbf{R}$.

Next we take an open subset $O_{1} \subset O$ such that $\left(O_{1}\right)_{\sigma_{t}} \subset O$ for some open interval

Now define again two functions

VI.1. Definition. For $A \in \mathscr{M}\left(O_{1}\right)$ and $B \in \mathscr{M}(O)^{\prime}$ put

$$
\begin{aligned}
& E(s, t)=\left(\Omega, \sigma_{s}^{o}(B) \sigma_{t}^{W}(A) \Omega\right), \\
& F(s, t)=\left(\Omega, \sigma_{t}^{W}(A) \sigma_{s}^{o}(B) \Omega\right),
\end{aligned}
$$

where $\sigma_{t}^{W}$ and $\sigma_{s}^{o}$ are the modular automorphisms of $\{\mathscr{M}(W), \Omega\}$ and $\{\mathscr{M}(O), \Omega\}$. These two functions are defined and continuous for $(s, t) \in \mathbf{R}^{2}$.

The functions are again boundary values of analytic functions. In detail we obtain:

VI.2. Lemma. (a) The function $E(s, t)$ is the boundary value of an analytic function $E(\sigma, \tau)$ holomorphic in:

$$
\left\{\sigma \in S\left(-\frac{1}{2}, 0\right)\right\} \times\left\{\tau \in S\left(-\frac{1}{2}, 0\right)\right\} .
$$

(the strip $S(.,$.$) has been defined in Lemma II.2)$ 
(b) The function $F(s, t)$ is the boundary value of an analytic function $F(\sigma, \tau)$ holomorphic in

$$
\left\{\sigma \in S\left(0, \frac{1}{2}\right)\right\} \times\left\{\tau \in S\left(0, \frac{1}{2}\right)\right\} .
$$

Proof. (a) $A \Omega$ is in the domain of $\left(\Delta^{W}\right)^{\frac{1}{2}}$, which implies that $\left(\Delta^{W}\right)^{i \tau} A \Omega$ is analytic in $-\frac{1}{2}<\mathfrak{I} m \tau<0$ and continuous on the boundary.

Since $B \in \mathscr{M}(O)^{\prime}$ it follows that $B^{*} \Omega$ belongs to the domain of $\left(\Delta^{O}\right)^{-1 / 2}$ which implies the analyticity of $\left(\Delta^{O}\right)^{i \sigma} B^{*} \Omega$ in the strip $0<\mathfrak{I} m \sigma<\frac{1}{2}$. Since $\Omega$ is reproduced by $\Delta^{O}$ we obtain $\sigma_{\sigma}^{o}\left(B^{*}\right) \Omega$ is analytic in $0<\mathfrak{I} m \sigma<\frac{1}{2}$. Taking now the adjoint we have to replace $\sigma$ by $\bar{\sigma}$. This leads to the stated result.

(b) The second statement can be derived from the first one by remarking

$$
F_{A, B}(\sigma, \tau)=\overline{E_{A^{*}, B^{*}}(\bar{\sigma}, \bar{\tau})}
$$

It remains to exploit the locality condition. For this it is important that $B$ belongs to $\mathscr{M}(O)^{\prime}$.

VI.3. Theorem. Under the assumptions made in the beginning of this section one has: $E(\sigma, \tau)$ and $F(\sigma, \tau)$ are two different branches of one holomorphic function $H(\sigma, \tau)$. This function is periodic along the diagonal in pure imaginary direction with periodicity $\iota$,

$$
H(\sigma+l, \tau+\imath)=H(\sigma, \tau)
$$

whenever $(\sigma, \tau)$ belongs to the domain of holomorphy of $H$. In particular one has

and

$$
E(\sigma, \tau)=H(\sigma, \tau), \quad \text { for } S\left(-\frac{1}{2}, 0\right) \times S\left(-\frac{1}{2}, 0\right),
$$

$$
F(\sigma, \tau)=H(\sigma, \tau), \quad \text { for } \quad S\left(0, \frac{1}{2}\right) \times S\left(0, \frac{1}{2}\right) .
$$

The functions $E$ and $F$ are connected as follows:

and

$$
E(s, t)=F(s, t), \quad \text { for } t \in I, \quad s \in \mathbf{R},
$$

$$
E\left(s-\frac{l}{2}, t-\frac{l}{2}\right)=F\left(s+\frac{l}{2}, t+\frac{l}{2}\right), \quad \text { for } \quad t \in \mathbf{R}, \quad s \in \mathbf{R} .
$$

The analytic continuation through the points $I \times \mathbf{R}$ respectively $\mathbf{R}^{2}$ are given by the local form of the edge of the wedge theorem.

Proof. Let $t \in I$ then $\sigma_{t}^{W}\left(O_{1}\right)$ belongs to $O$ and hence $\sigma_{t}^{W}(A)$ and $\sigma_{s}^{O}(B)$ commute for $s \in \mathbf{R}$. Therefore, we get by the edge of the wedge theorem an analytic connection between $E(\sigma, \tau)$ and $F(\sigma, \tau)$.

Next compute $E\left(s-\frac{l}{2}, t-\frac{l}{2}\right)$. This gives:

$$
\begin{aligned}
E\left(s-\frac{l}{2}, t-\frac{l}{2}\right) & =\left(\Omega, \sigma_{\left(s-\frac{l}{2}\right)}^{o}(B) \sigma_{\left(t-\frac{l}{2}\right)}^{W}(A) \Omega\right) \\
& =\left(\sigma_{\left(s-\frac{l}{2}\right)}^{o}\left(B^{*}\right) \Omega, \sigma_{\left(t-\frac{l}{2}\right)}^{W}(A) \Omega\right)
\end{aligned}
$$


Translation Group and Modular Automorphisms for Local Regions

$$
\begin{aligned}
& =\left(\left(\Delta^{o}\right)^{-1 / 2} \sigma_{s}^{o}\left(B^{*}\right) \Omega,\left(\Delta^{W}\right)^{1 / 2} \sigma_{t}^{W}(A) \Omega\right) \\
& =\left(J^{o} \sigma_{s}^{o}(B) \Omega, J^{W} \sigma_{t}^{W}\left(A^{*}\right) \Omega\right) \\
& =\left(j^{o}\left(\sigma_{s}^{o}(B)\right) \Omega, j^{W}\left(\sigma_{t}^{W}\left(A^{*}\right)\right) \Omega\right) \\
& =\left(\Omega, j^{o}\left(\sigma_{s}^{o}\left(B^{*}\right)\right) j^{W}\left(\sigma_{t}^{W}\left(A^{*}\right)\right) \Omega\right) .
\end{aligned}
$$

Here $J^{O}$ respectively $J^{W}$ denote the modular conjugations for $\mathscr{M}(O)$ respectively $\mathscr{M}(W)$. Now we compute $F\left(s+\frac{l}{2}, t+\frac{l}{2}\right)$ :

$$
\begin{aligned}
& F\left(s+\frac{l}{2}, t+\frac{l}{2}\right)=\left(\Omega, \sigma_{\left(t-\frac{l}{2}\right)}^{\left.(A) \sigma_{\left(s-\frac{l}{2}\right)}^{o}(B) \Omega\right)}\right. \\
& =\left(\sigma_{\left(t-\frac{l}{2}\right)}^{W}\left(A^{*}\right) \Omega, \sigma_{\left(s-\frac{l}{2}\right)}^{o}(B) \Omega\right) \\
& =\left(\left(\Delta^{W}\right)^{1 / 2} \sigma_{t}^{W}\left(A^{*}\right) \Omega,\left(\Delta^{O}\right)^{-1 / 2} \sigma_{s}^{o}(B) \Omega\right) \\
& =\left(J^{W} \sigma_{t}^{W}(A) \Omega, J^{o} \sigma_{s}^{o}\left(B^{*}\right) \Omega\right) \\
& =\left(j^{W}\left(\sigma_{t}^{W}(A)\right) \Omega, j^{o}\left(\sigma_{s}^{o}\left(B^{*}\right)\right) \Omega\right) \\
& =\left(\Omega, j^{W}\left(\sigma_{t}^{W}\left(A^{*}\right)\right) j^{o}\left(\sigma_{s}^{o}\left(B^{*}\right)\right):\right) \text {. }
\end{aligned}
$$

$A$ belongs to $\mathscr{M}\left(O_{1}\right)$ and hence $\sigma_{t}^{W}\left(A^{*}\right)$ is an element of $\mathscr{M}(W)$. This implies $j^{W}\left(\sigma_{t}^{W}\left(A^{*}\right)\right) \in \mathscr{M}(W)^{\prime}$. Similar we have $B \in \mathscr{M}(O)^{\prime}$ which implies $\sigma_{s}^{O}\left(B^{*}\right) \in \mathscr{M}(O)^{\prime}$ and consequently $j^{o}\left(\sigma_{s}^{o}\left(B^{*}\right)\right) \in \mathscr{M}(O)$. Since now $\mathscr{M}(O) \subset \mathscr{M}(W)$ is follows that $j^{W}\left(\sigma_{t}^{W}\left(A^{*}\right)\right)$ and $j^{O}\left(\sigma_{s}^{o}\left(B^{*}\right)\right)$ commute for all values of $s$ and $t$ and therefore:

$$
E\left(s-\frac{l}{2}, t-\frac{l}{2}\right)=F\left(s+\frac{l}{2}, t+\frac{l}{2}\right), \quad \text { for } \quad(s, t) \in \mathbf{R}^{2} .
$$

This proves the theorem.

\section{Problems of Causality}

We are going back to the situation described in Sect. III because here the implications of local commutativity are more direct. We had a domain $V$ and a double cone $D$, an operator $A \in \mathscr{M}(V)$ and an operator $B \in \mathscr{M}(D)$. Then local commutativity implies that $\alpha_{a}(B)$ and $\sigma_{t}(A)$ commute for $a \in G_{2} .\left(\sigma_{t}\right.$ is the modular automorphism of the pair $\{\mathscr{M}(V), \Omega\}$.) Let us now take a domain $O_{1} \subset V$ small enough such that also $O_{1}+a \subset V$ for a in some open neighbourhood $N$ of the origin in $\mathbf{R}^{d}$, and let us take $A \in \mathscr{M}\left(O_{1}\right)$. Define

$$
G_{2}^{(1)}=\left\{a \in \mathbf{R}^{d}, D+a \in O_{1}^{\prime}\right\} .
$$

We obviously have $G_{2} \subset G_{2}^{(1)}$. Looking at the functions

$$
E(a, 0)=\left(\Omega, \alpha_{a}(B) A \Omega\right)
$$


and

we see that

$$
F(a, 0)=\left(\Omega, A \alpha_{a}(B) \Omega\right)
$$

$$
E(a, 0)=F(a, 0) \text { for } a \in G_{2}^{(1)} .
$$

Moreover, the functions $E(a, 0)$ and $F(a, 0)$ are boundary values of analytic functions holomorphic in the tubes $T^{-}$respectively $T^{+}$, and by the edge of the wedge theorem also in $G_{2}^{(1)}$.

There are now two possibilities. Either this extra analyticity is purely accidental. In this case every modular automorphism $\sigma_{t}$ spreads an operator, localized in a subregion, into the whole region. The other possibility is that the analyticity of the functions $E(a, 0), F(a, 0)$, in the one variable, is a consequence of the analyticity of the functions $E(a, t), F(a, 0)$ in both variables.

The second situation appears rarely. The reason for this is the necessity of sharp localization of the state in momentum space. If one has the vacuum state the examples of the wedge domain and the double cone for the massless free field show that one has localization properties for the modular automorphisms. Since all other states of finite energy are obtained by smearing improper states of sharp momentum it is clear that one loses by smearing the local properties of the modular transformations. However, the modular operator belonging to a given bounded region is not a unique object, but it is also connected to the cyclic and separating vector one has chosen. This implies that not a single modular operator but the whole family is of interest. Therefore, one should look at the possibility of extracting from this family an object which is free from the accidental choice of the cyclic and separating vector and which might act in a local fashion.

One should be aware that there might be an obstruction for this project. If we look at the algebra of the free field with the vacuum as cyclic and separating vector then in the massless case one has modular automorphisms which act locally. However, for the massive free fields this is no longer the case. For the wedge domain one has locally acting modular automorphisms even for interacting fields. The source for these differences in behaviour is up to now not well understood.

We are now coming to the problem of interpreting the modular operator as a function of some form of local energy. In order to obtain a result of this kind it has to be shown that one finds the Hamiltonian when going with the radius of the double cone to infinity. First we remark that the expression $\alpha_{t}(A) \Omega$, with $\alpha_{t}$ as time translation, is a vector valued function holomorphic in the upper half plane. The expression $\sigma_{t}(A) \Omega$, where $\sigma_{t}$ is the modular automorphism of the double cone $D$, is only analytic in the strip $S(-1 / 2,0)$. Hence, one has to scale the variable when going with $D$ to infinity. If one succeeds to prove that after proper rescaling the limit

$$
\lim _{D \rightarrow \infty} \sigma_{s(t)}^{D}
$$

converges, one faces the problem of showing that the limiting automorphisms act locally and represent the time translations. One way of proving this needs two steps. If we consider the vacuum sector and the vacuum as cyclic and separating vector the modular group of the wedge domain is known. Hence, if the edge of the wedge tends to spacelike infinity, it is easily shown that one obtains, after correct scaling, the time translations. Using next the result of Sect. IV, one should be able to show that 
the modular automorphisms of the wedge domain and the double cones converge to each other. The reason is the periodicity along the diagonal. Periodic functions which are bounded on the boundaries are also bounded in the complex. In the limit one obtains after scaling a function entire analytic and bounded along the diagonal. This shows that the corresponding expectation values depend only on one variable. This is equivalent to the statement that the corresponding automorphism groups coincide. Details will be presented in a forthcoming paper.

There is another aspect of the result obtained in Sect. IV. K. Fredenhagen [7] was able to compare the modular operator of the wedge domain with that of the double cone provided it is in the wedge domain and its closure has one point in common with the edge of the wedge. One should be able to obtain similar results for an arbitrary double cone inside the wedge by using the periodicity of the expectation value. The main problem here will be a good estimate of the envelop of holomorphy.

\section{References}

1. Bisognano, J., Wichmann, E.H.: On the duality for a hermitian scalar field. J. Math. Phys. 16, 985-1007 (1975)

2. Borchers, H.-J.: On the converse of the Reeh-Schlieder theorem. Commun. Math. Phys. 10, 269-273 (1968)

3. Brehmermann, H.J., Oehme, R., Taylor, J.D.: Proof of dispersion relation in quantized field theories. Phys. Rev. 109, 2178-2190 (1958)

4. Buchholz, D., D'Antoni, C., Longo, R.: Nuclear maps and modular structure. II. Applications to quantum field theory. Commun. Math. Phys. 129, 115-138 (1990)

5. Buchholz, D., and Junglas, P.: On the existence of equilibrium states in local quantum field theory. Commun. Math. Phys. 121, 255-270 (1989)

6. Buchholz, D., Wichman, E.H.: Causal independence and the energy level density of states in local quantum field theory. Commun. Math. Phys. 106, 321-344 (1986)

7. Fredenhagen, K.: On the modular structure of local algebras of observables. Commun. Math. Phys. 97, 79-89 (1985)

8. Hislop, P.D., Longo, R.: Modular structure of the local algebra associated with a free massless scalar field theory. Commun. Math. Phys. 84, 71-85 (1982)

9. Reeh, H., Schlieder, S. : Bemerkungen zur Unitäräquivalenz von Lorentzinvarianten Feldern. Il Nuovo Cimento 22, 1051-1068 (1961)

10. Takesaki, M.: Tomita's theory of modular Hilbert algebras and its applications, Lecture Notes in Mathematics. Berlin, Heidelberg, New York: Springer 1970

11. Tomita, M. : Standard forms of von Neumann algebras. Fifth functional analysis symposium of Math. Soc. of Japan, Sendai

Communicated by A. Jaffe

Received April 3, 1990 
